RELATO DE CASO

ISSN 1677-5090

(C) 2019 Revista de Ciências Médicas e Biológicas

\title{
Escleroterapia como tratamento conservador para hemangioma oral: relato de caso
}

\author{
Sclerotherapy as a conservative approach for oral hemangioma: case report
}

\author{
Jéssica Alves Gomes', Luciana Maria Pedreira Ramalho* \\ ${ }^{1}$ Mestranda do Programa de Pós-graduação em Processos Interativos dos Órgãos e Sistemas, do Instituto de \\ Ciências da Saúde, da Universidade Federal da Bahia. ${ }^{2}$ Doutora em Odontologia, pela Pontífica Universidade \\ Católica do Rio Grande do Sul, professora titular da Faculdade de Odontologia, da Universidade Federal da Bahia.
}

\begin{abstract}
Resumo
Introdução: o hemangioma é um tumor benigno vascular de origem endotelial, definido por crescimento anormal dos vasos sanguíneos. Essa lesão pode atingir qualquer parte do corpo, porém a região de cabeça e pescoço tem uma incidência de $60 \%$ dos casos; na cavidade oral, ela pode se apresentar na língua, na mucosa bucal, no palato, nos lábios. O tratamento é multimodal, incluindo laser, crioterapia, excisão cirúrgica, agentes quimioterápicos e escleroterapia. Objetivo: apresentar um relato de caso de escleroterapia em hemangioma de dorso de língua, tratado com dose única de oleato de monoetanolamina. Metodologia: paciente do sexo feminino, 35 anos, branca, apresentou-se a um consultório particular queixando-se de lesão pigmentada na língua, assintomática, notada após contato com aparelho ortodôntico lingual. Ao exame clínico intrabucal foi observada lesão nódulo papular, de coloração vermelha, localização em dorso de língua, com $0.5 \mathrm{~cm}$ de diâmetro e inserção séssil com diagnóstico de hemangioma oral. Tratado em dose única com oleato de monoetanolamina a $0.4 \mathrm{ml}$ na proporção de $50 \%$, diluído na solução anestésica local de lidocaína com adrenalina $1 \backslash 100.000$ no centro da lesão. Resultados: a lesão apresentou regressão total da lesão e sem recidiva após uma sessão do tratamento. Conclusão: escleroterapia é um tratamento conservador, eficiente e com resultado estético positivo, nos casos de hemangioma, porém vale ressaltar que esta deve ser devidamente indicada, analisando sempre as suas limitações e os seus benefícios. Palavras-chaves: Hemangioma. Escleroterapia. Boca. Língua.
\end{abstract}

\begin{abstract}
Introduction: hemangioma is a benign vascular tumor of endothelial origin defined by abnormal growth of blood vessels. This injury can affect any part of the body, but the head and neck region has an incidence of $60 \%$ of cases. In the oral cavity it may be present in the tongue, buccal mucosa, palate, lips. Treatment is multimodal including laser, cryotherapy, surgical excision, chemotherapeutic agents and sclerotherapy. Objective: to present a case report of sclerotherapy in tongue dorsal hemangioma treated with a single dose of monoethanolamine oleate. Methodology: a 35-year-old white female patient presented to a private practice complaining of asymptomatic pigmented tongue injury noted after contact with the lingual orthodontic appliance. Clinical examination revealed a red papular nodule lesion, located on the back of the tongue, $0.5 \mathrm{~cm}$ in diameter and sessile insertion diagnosed with Oral Hemangioma. The nodule was treated in the lesion center with a single dose of $0.4 \mathrm{ml}$ monoethanolamine oleate, at a proportion of $50 \%$ dilution in $1 \mid 100,000$ local anesthetic solution of lidocaine with adrenaline. Results: total regression of the lesion and no recurrence was presented after 01 treatment session. Conclusion: sclerotherapy is a conservative treatment, efficient and with positive aesthetic result in cases of Hemangioma, but it is worth mentioning that it should be properly indicated always considering its limitations and its benefits. Keywords: Hemangioma. Sclerotherapy. Mouth. Tongue.
\end{abstract}

\section{INTRODUÇÃO}

As anomalias vasculares podem ser divididas em tumores e malformações vasculares (FOWELL et al., 2016; KATO et al., 2019). O hemangioma é um tumor benigno vascular de origem endotelial, definido por crescimento anormal dos vasos sanguíneos (ABDYLI et al., 2016; CALIENTO et al., 2014; MANDÚ et al., 2013; PALMA et al., 2016; PEREIRA; CARIRI, 2018; DA SILVA et al., 2014; ROCHA et al., 2014). Infecções, traumas, alterações hormonais e

Correspondente/Corresponding: *Luciana Maria Pedreira Ramalho - End: Avenida Araújo Pinho, 62 Canela CEP:40110-150 - E-mail: lucianaramalho@uol.com.br -Tel:(71) 99904-3168 condições ligadas à gestação são fatores que podem estar associados com a sua etiologia (CALIENTO et al., 2014; DA ROCHA et al., 2014). Crianças logo após o nascimento ou na primeira infância e mulheres são os mais acometidos por essa patologia (CALIENTO et al., 2014; KATO et al., 2019; KORVIPATI et al., 2016; QUEIROZ et al., 2014; DA ROCHA et al., 2014). Essa lesão pode atingir qualquer parte do corpo, porém a região de cabeça e pescoço tem uma incidência de $60 \%$ dos casos (MANDÚ et al., 2013; PEREIRA; CARIRI, 2018). Na cavidade oral, o hemangioma é diagnosticado com maior frequencia na língua, na mucosa bucal, no palato e nos lábios (MANDÚ et al., 2013; QUEIROZ et al., 2014). A condição intraóssea é rara (ABDYLI et al., 2016; CALIENTO et al., 2014; FERNANDES et al., 2018). 
Normalmente, os hemangiomas são assintomáticos e apresentam uma larga escala de tamanho, sendo que quando maiores podem causar até assimetria facial. A sua coloração depende da profundidade da lesão, do grau de congestão vascular e da sua localização, variando, assim, de vermelho a roxo. A lesão pode apresentar consistência elástica ou fibrosa, de base séssil ou pediculada, de superfície lisa ou irregular, forma plana ou elevada, bordas definidas, sendo classificada como mácula, pápula, nódulo ou tumor. Quando submetida à pressão, pode apresentar palidez devido à vasoconstricção (ABDYLI et al., 2016; CALIENTO et al., 2014; FERNANDES et al., 2018; QUEIROZ et al., 2014).

O diagnóstico clínico é primordial, baseado na história e características da lesão, porém, com efeito de diferenciação de outras lesões orais, como melanoma oral, melanose racial, melanose induzida por tabaco e drogas, Sarcoma de Kaposi, cisto de retenção, ganulomapiogênico e mucocele. As manobras semiotécnicas, como punção aspirativa, compressão digital e vitropressão, e os exames complementares, como diascopia, biópsia e exames de imagem, podem ser realizados para diagnóstico ( ABDYLI et al., 2016; FERNANDES et al., 2018; SILVA et al., 2014; QUEIROZ et al., 2014).

Existem algumas alternativas para o tratamento do hemangioma, como o laser, a crioterapia, excisão cirúrgica, corticoides intra-lesionais, embolização, agentes quimioterápicos e escleroterapia (CORRÊA et al., 2007; FERNANDES et al., 2018; KATO et al., 2019; MANDÚ et al., 2013; PALMA et al., 2016; DA SILVA et al., 2014; ROCHA et al., 2014). A escolha do tratamento depende da localização, do tamanho, grau de invasão, hemodinâmica da lesão, idade do paciente e possibilidade da realização da técnica (KATO et al., 2019).

Escleroterapia é um tratamento não cirúrgico, utilizado para diminuir ou eliminar vasos pequenos ou veias. A escleroterapia é um método efetivo, conservador, sem necessidade de incisão cirúrgica, de baixo custo. Através dele, obtêm-se resultados positivos em lesões pequenas de importância clínica funcional e estética (JOHANN et al., 2005; MANDÚ et al., 2013; QUEIROZ et al., 2014; DA ROCHA et al., 2014).

O objetivo deste trabalho é apresentar um relato de caso de hemangioma em dorso de língua, tratado com dose única de oleato de monoetanolamina, discutindo a sua aplicabilidade clínica.

\section{RELATO DE CASO}

Paciente do sexo feminino, 35 anos, branca, apresentou-se a um consultório particular, queixando-se de lesão pigmentada na língua, assintomática. Relatou ter sido alertada pelo cirurgião-dentista há três meses e encaminhada para o especialista. Disse observar crescimento da lesão no local que entrava em contato com aparelho ortodôntico lingual. Ao exame clínico extrabucal, não foram observadas alterações. Ao exame clínico intrabucal, foi observada lesão nódulo papular, de coloração vermelha, localização em dorso de língua, com $0.5 \mathrm{~cm}$ de diâmetro e inserção séssil. O diagnóstico clínico foi de hemangioma oral.

Por tratar-se de uma lesão com tamanho reduzido, a proposta apresentada à paciente foi a escleroterapia. $\mathrm{O}$ agente esclerosante eleito foi o oleato de monoetanolamina, que atua por irritação da camada íntima do endotélio venoso, produzindo uma resposta inflamatória, de acordo com a dose utilizada. Por consequência, há uma fibrose vascular levando à oclusão. Ele também produz uma reação inflamatória extravascular, a depender da dose utilizada, por conta da sua difusão rápida através da parede da veia.

No momento da aplicação, procedeu-se à limpeza da área com clorexidina $0,12 \%$; posteriormente, injetou-se no centro da lesão $0,4 \mathrm{ml}$ da solução esclerosante na proporção de $50 \%$, diluída na solução anestésica local de lidocaína com adrenalina $1 \backslash 100.000$, com o auxílio de seringa de insulina. Em seguida, promoveu-se a vasoconstricção da área através da aplicação de gelo por 2 minutos. A paciente foi então orientada a retornar para reavaliação em 30 dias, considerando que ela residia no interior do estado. Não foi necessária uma segunda aplicação diante a completa resolução da lesão, caso que permaneceu até oito meses após a aplicação. A paciente relatou edema e desconforto de leve intensidade nas 48 horas subsequentes à aplicação, mas não foi necessário o controle medicamentoso.

Figura 1 - Aspecto clínico na primeira consulta.

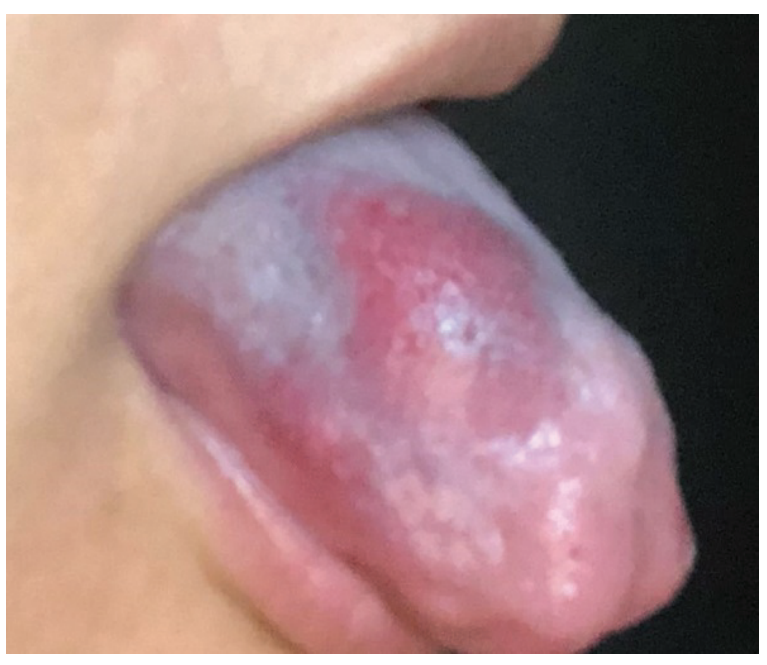

Fonte: As autoras. 
Figura 2 - Aspecto clínico nas 48 horas subsequentes à aplicação.

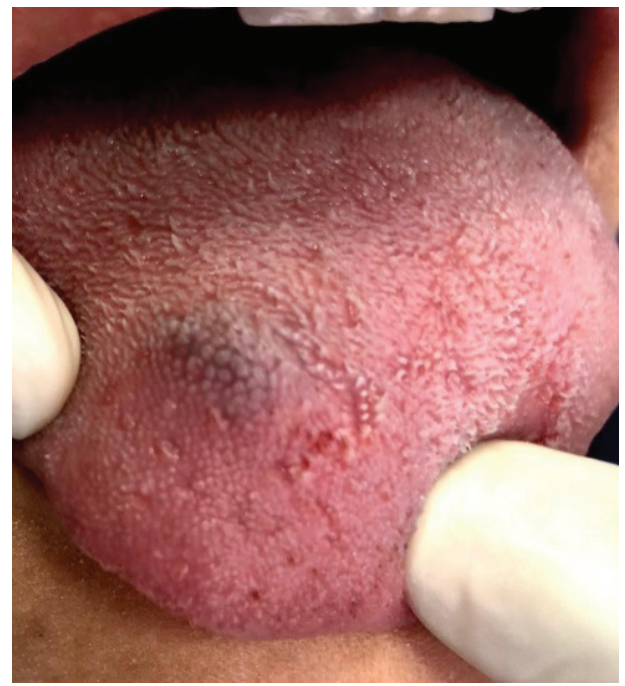

Fonte: As autoras.

Figura 3 - Aspecto clínico após oito meses da aplicação.

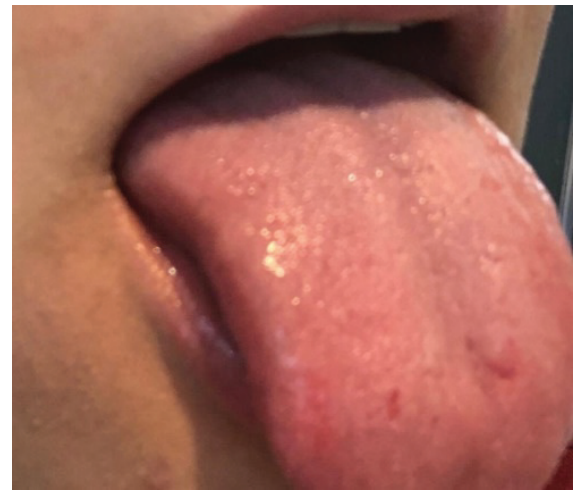

Fonte: As autoras

\section{DISCUSSÃO}

O hemangioma se apresenta com maior incidência em pessoas do sexo feminino, na proporção de 3:1 ( KORVIPATI et al., 2016; ROCHA et al., 2014). A lesão pode se estender por toda a extremidade corpórea, porém, quando afeta a cavidade bucal os locais de maior acometimento são língua, mucosa jugal e palato (ABDYLI et al., 2016; CORRÊA et al., 2007; ROCHA et al., 2014). Esse relato de caso reforça os achados da literatura, uma vez que a paciente é do sexo feminino e a localização da lesão é em língua. Embora tenha mais incidência na infância (CALIENTO et al., 2014), pode ser encontrado em qualquer idade (ROCHA et al., 2014), como o presente caso, que se relaciona à fase adulta.

O surgimento da lesão pode estar associado a fator congênito ou traumático (ROCHA et al., 2014), o que corrobora o relato da paciente de que observou o crescimento da lesão no local de contato com aparelho ortodôntico lingual, um suposto fator traumático.
Os aspectos clínicos da lesão se manifestam de acordo com a sua profundidade e o seu local (ABDYLI et al., 2016). Dessa maneira a sua coloração pode variar do vermelho ao roxo. O tamanho é variável de milímetros à centímetros, podendo ocorrer até assimetrias faciais (FERNANDES et al., 2018). A consistência pode ser elástica ou fibrosa, a superfície pode ser lisa ou irregular. As bordas são definidas, o que auxilia na classificação entre mácula, pápula, nódulo ou tumor (ABDYLI et al., 2016; CALIENTO et al., 2014; FERNANDES et al., 2018; QUEIROZ et al., 2014). Neste caso, por se tratar de uma lesão pouco profunda, sua coloração se apresentava arroxeada. Além de se mostrar com poucos milímetros, ela se localizava em dorso de língua, o que não favorecia uma assimetria facial.

A excisão cirúrgica para hemangiomas periféricos e para lesões de tamanho reduzido e circunscritas é o tratamento de referência. Porém, devido a sangramentos excessivos no pós-operatório, surgiram as demais modalidades de tratamento que incluem a escleroterapia (ABDYLl et al., 2016). Outras complicações que podem ocorrer no tratamento de excisão cirúrgica são na cicatrização e na disfunção de órgãos e tecidos (SILVA et al., 2014).

Na escleroterapia, o mecanismo de ação se conclui mediante uma resposta inflamatória, envolvendo a troca do componente vascular por um tecido fibrótico. A dose, a concentração do agente esclerosante e o modo de aplicação não são propriamente padronizados, devendo, assim, respeitar a individualidade funcional e morfológica da lesão (FERNANDES et al., 2018), como tamanho, envolvimento de estruturas adjacentes, profundidade, evolução da lesão e localização (ABDYLI et al., 2016). Os pontos de importância para a escolha do tratamento do presente estudo foram: a lesão apresentar tamanho reduzido, sem envolvimento de estruturas adjacentes e pouco profundas.

A fim de evitar necrose da mucosa bucal, a aplicação deve ser realizada no centro da lesão e de maneira profunda, ao invés de superficial (CALIENTO et al., 2014). A quantidade da dose do fármaco está associada com as reações adversas da aplicação (SILVA et al., 2014). As dimensões da lesão vão direcionar a dose a ser aplicada, porém, geralmente não se deve exceder $2 \mathrm{ml}$ (QUEIROZ et al., 2014). Neste caso, foram utilizados $0,4 \mathrm{ml}$ da solução esclerosante e a aplicação foi realizada no centro da lesão, o que pode ter auxiliado na ausência de necrose tecidual.

Quando instituída a melhor forma de tratamento diante do caso clínico, os hemangiomas costumam não recidivar ou sofrer malignização (MANDÚ et al., 2013). Como se pode observar no presente caso, houve uma regressão total da lesão e sem recidiva. Quanto à escleroterapia, estão descritos na literatura alguns agentes esclerosantes, como morruato de sódio $5 \%$, psilato de sódio, etanol 95\%, solução hipertônica de glicose (50 e $75 \%)$, água quente, álcool $95 \%$, polidocanol $1 \%$, tetradecil sulfato de sódio e oleato de monoetanolamina (2,5 e 5\%) (KATO et al., 2019; ROCHA et al., 2014). Os dois primeiros passaram a não ser utilizados por apresentarem algumas 
reações adversas, como alergias e dor local. Os dois últimos são os mais utilizados atualmente (ROCHA et al., 2014), recaindo no oletato de monoetanolamina a escolha para o tratamento aqui relatado.

\section{CONCLUSÃO}

No presente caso, observou-se a regressão total da lesão após escleroterapia, com dose única de oleato de monoetanolamina, e sem recidiva após um ano da aplicação. Dessa forma apresentamos a escleroterapia como proposta terapêutica conservadora, eficiente e com resultado estético positivo, nos casos de hemangioma. Vale ressaltar que, assim como em qualquer terapia, esta deve ser devidamente indicada, analisando sempre as suas limitações e os seus benefícios.

\section{ASPECTOS ÉTICOS}

Este relato de caso foi aprovado pelo Comitê de Ética em Pesquisa, da Faculdade de Odontologia, da Universidade Federal da Bahia, sob o Parecer n. 3.509.842.

\section{REFERÊNCIAS}

ABDYLI, R. A. et al. Sclerotherapy of Intraoral Superficial Hemangioma. Case rep. dent., Bethesda, v. 2016, p. 1-5, 2016.

CALIENTO, R. et al. Tratamento de hemangioma por escleroterapia em aplicação única. Rev. cir. Traumatol. buco-maxilo-fac., Camurugibe, Pe, v. 14, n. 3, p. 27-32, 2014.

CORRÊA, P. H. et al. Prevalence of oral hemangioma, vascular malformation and varix in a Brazilian population. Braz oral res., São Paulo, v. 21, n. 1, p. 40-45, 2007.

FERNANDES, D. T. et al. Benign oral vascular lesions treated by sclerotherapy with ethanolamine oleate: A retrospective study of 43 patients. Med. oral pathol. oral cir. bucal, Estados Unidos, v. 23, n. 2, p. 180-187, 2018.

FOWELL, C. et al. Venous malformations of the head and neck: current concepts in management. $\mathrm{Br}$ j. oral maxillofac. surg., Edinburg, v. 55, n. 1, p. 1-7, 2016.

JOHANN, A. C. B. R. et al. Sclerotherapy of benign oral vascular lesion with ethanolamine oleate: An open clinical trial with 30 lesions. Oral Surg. Oral Med. Oral Pathol. Oral Radiol. Endod., St Louis, v. 100, n. 5, p. 579-584, 2005.

KATO, C. de N. A. de O. et al. Experience with 5\% ethanolamine oleate for sclerotherapy of oral vascular anomalies: A cohort of 15 consecutive patients. J. craniomaxillofac. surg., New York, v. 47, n. 1, p. 106-111, 2019.

KORVIPATI, N. et al. Sclerotherapy for oral hemangioma. J. Indian Acad. Oral Med. Radiol., India, v. 28, n. 2, p. 188-190, 2016.

MANDÚ, A. L. C. et al. Escleroterapia de hemangioma: relato de caso. Rev. cir. Traumatol. buco-maxilo-fac., Camurugibe, Pe, v. 13, n. 1, p. 71-76, 2013.

PALMA, F. R. et al. Escleroterapia de hemangioma oral: relato de caso. Rev. Salusvita, Bauru, v. 35, n. 1, p. 85-93, 2016.

PEREIRA, E. A.; CARIRI, T. F. A. Escleroterapia em lesões vasculares de boca: relato de dois casos clínicos. RFO - UPF, Passo Fundo, v. 23, n. 3 , p. 315-321, 2018.

QUEIROZ, S. I. M. L. et al. Tratamento de hemangioma oral com escleroterapia: relato de caso. J. vasc. bras., Rio de Janeiro, v. 13, n. 3 , p. 249-253, 2014.

ROCHA, A. B. M. et al. Escleroterapia de hemangioma em borda lateral de língua: relato de caso. Rev. Ciênc. Méd. Biol., Salvador, v. 13, n. 3, p. 398-402, 2014.

SILVA, W. B. et al. Oral capillary hemangioma: A clinical protocol of diagnosis and treatment in adults. Oral Maxillofac. Surg., Springer, $v$. 18, n. 4, p. 431-437, 2014.

Submetido em: 04/11/2019

Aceito em: 11/11/2019 